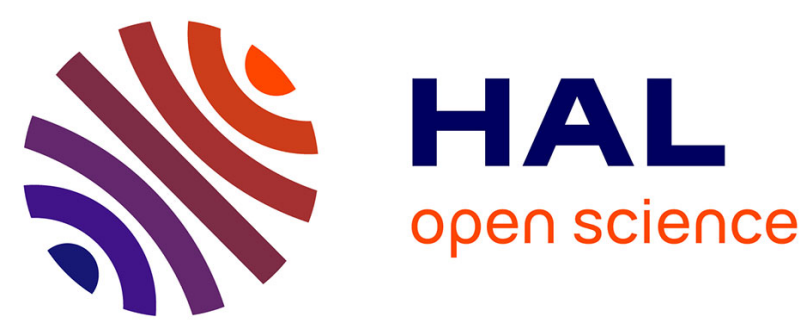

\title{
EEG source analysis in obsessive-compulsive disorder
}

Jana Kopřivová, Marco Congedo, Jiří Horáček, Ján Praško, Michal Raszka,

Martin Brunovský, Barbora Kohútová, Cyril Höschl

\section{To cite this version:}

Jana Kopřivová, Marco Congedo, Jiří Horáček, Ján Praško, Michal Raszka, et al.. EEG source analysis in obsessive-compulsive disorder. Clinical Neurophysiology, 2011, 122 (9), pp.1735-1743. 10.1016/j.clinph.2011.01.051 . hal-00620687

\section{HAL Id: hal-00620687 \\ https://hal.science/hal-00620687}

Submitted on 8 Sep 2011

HAL is a multi-disciplinary open access archive for the deposit and dissemination of scientific research documents, whether they are published or not. The documents may come from teaching and research institutions in France or abroad, or from public or private research centers.
L'archive ouverte pluridisciplinaire HAL, est destinée au dépôt et à la diffusion de documents scientifiques de niveau recherche, publiés ou non, émanant des établissements d'enseignement et de recherche français ou étrangers, des laboratoires publics ou privés. 
Title: EEG source analysis in obsessive-compulsive disorder

Authors: Jana Kopřivová a, b , Marco Congedo c, Jiří Horáček ${ }^{a, b}$, Ján Praško ${ }^{a, d}$, Michal Raszka ${ }^{a, b}$, Martin Brunovský a, b Barbora Kohútová $^{\mathrm{a}, \mathrm{b}}$, Cyril Höschl ${ }^{\mathrm{a}, \mathrm{b}}$

${ }^{a}$ Prague Psychiatric Centre, Prague, Czech Republic

${ }^{b}$ Third Faculty of Medicine, Charles University, Prague, Czech Republic

${ }^{c}$ Team ViBS (Vision and Brain Signal Processing), GIPSA-Iab, CNRS (Centre National de la Recherche Scientifique), Grenoble University, France

${ }^{d}$ Department of Psychiatry, University Hospital Olomouc, Czech Republic

Corresponding author: Jana Kopřivová, Prague Psychiatric Centre, Ústavní 91, 18103 Prague 8, Czech Republic Tel. +420266003 361, Fax: +420266003 366

E-mail address: koprivova@pcp.If3.cuni.cz

Key-words: obsessive-compulsive disorder (OCD); standardized low-resolution electromagnetic tomography (sLORETA); independent component analysis (ICA); medial frontal cortex

Acknowledgements: This work was supported by the grant IGA NS 9751-3/2008 provided by the Ministry of Health of the Czech Republic.

\section{Abstract}

OBJECTIVE: The goal of this study was to assess the activity of intracortical EEG sources in patients with OCD. METHODS: We compared resting state EEG from 50 OCD patients and 50 matched controls using standardized low-resolution electromagnetic tomography (sLORETA) and normative independent component analysis (NICA). Data were analyzed with 1 $\mathrm{Hz}$ frequency resolution. Group ICA was used to separate 7 independent components from the control group data. The resulting weights and norms served to derive the same components from the OCD group and to compare their power with controls. RESULTS: In OCD, sLORETA indicated low-frequency power excess $(2-6 \mathrm{~Hz})$ in the medial frontal cortex, whereas group ICA showed increased low-frequency power in a component reflecting the activity of subgenual anterior cingulate, adjacent limbic structures and to a lesser extent also of lateral frontal cortex. CONCLUSIONS: Both methods provided evidence for medial frontal hyperactivation in OCD. SIGNIFICANCE: Our study is the first to use normative ICA in a clinical sample and indicates its potential utility as a diagnostic tool. The findings provide consistent results based on EEG source localization in $O C D$ and are of practical interest for therapeutic interventions.

\section{INTRODUCTION}

Obsessive-compulsive disorder (OCD) is a common neuropsychiatric disorder marked by recurrent intrusive thoughts (obsessions) and repetitive behaviours (compulsions) with a prevalence of 2-3\% (Karno et al., 1988). Neuroimaging studies provide evidence for the involvement of cortico-striatal circuits in OCD pathophysiology (Aouizerate et al., 2004). The traditional and most widely accepted model, supported by a large body of scientific evidence, postulates a hyperactive orbitofronto-striatal circuit including orbitofrontal and cingulate cortex, ventral striatum, ventral pallidum, mediodorsal thalamus, hippocampus and amygdala (Menzies et al., 2008a). However, accumulating evidence from various methodological approaches suggests that OCD is mediated by more widely distributed neural networks including also dorsal brain regions such as dorsolateral prefrontal cortex (Gu et al., 2008; Remijnse et al., 2006; van den Heuvel et al., 2005), parietal cortex (Menzies et al., 2008b) or cerebellum (Nabeyama et al., 2008; van den Heuvel et al., 2009). A recent multimodal review and meta-analytic study by Menzies et al. (2008a) proposed a more comprehensive OCD model including two relatively segregated fronto-striatal networks: affective orbitofronto-striatal loop and dorsolateral prefrontostriatal loop which also includes parietal and lateral prefrontal cortex and subserves spatial and attentional functions. Aberrant functioning and imbalanced interactions between frontostriatal networks might explain clinical OCD symptoms and neuropsychological deficits such as excessive perception of error (Ullsperger and von Cramon, 2006), abnormal reward processing (Remijnse et al., 2006), cognitive and behavioural inflexibility (Gu et al., 2008) and difficulty to inhibit prepotent responses (Roth et al., 2007).

Electroencephalographic (EEG) studies based on quantitative analysis reported abnormalities registered at frontal or frontotemporal electrode sites in OCD (e.g. Karadag et al., 2003; Pogarell et al., 2006; Prichep et al., 1993). However, only limited attention has been paid to the localization of generators of the aberrant EEG activity in OCD. EEG sources have been investigated in relation to treatment response (Bolwig et al., 2007; Fontenelle et al., 2006) and in subjects with obsessive-compulsive symptoms (Sherlin and Congedo, 2005) and recently also in drug naïve obsessive-compulsive patients (Velikova et al., 2010). All studies using low- 
resolution electromagnetic tomography in OCD (Fontenelle et al., 2006; Sherlin and Congedo, 2005; Velikova et al., 2010) reported significant results in anterior cingulate (ACC). In addition, abnormal ACC functioning in OCD has been supported by evoked-potential EEG research that revealed an enhanced error-related negativity (ERN) in OCD patients. ERN is generated after erroneous response or in conflict situations and reflects activity of the performance monitoring system in OCD (Endrass et al., 2008). Its source has been localized near to dorsal ACC (Dehaene et al., 1994) using electrophysiological inverse solution methods as well as simultaneous EEG and fMRI recording (Debener et al., 2005). Functional magnetic resonance studies confirmed the overactivity of the performance monitoring system in OCD and its link with ACC and medial frontal cortex hyperactivation (Fitzgerald et al., 2005; Ursu et al., 2003). It has been hypothesized that an overactive performance monitoring system generates a feeling that something is wrong or not just right and triggers compulsive behaviour, thus providing a possible explanation how brain abnormalities translate in clinical symptoms of OCD (Maltby et al., 2005).

Our study aimed to compare resting state current density power in intracortical sources between OCD patients and control subjects as well as between unmedicated patients and patients medicated with selective serotonin reuptake inhibitors (SSRIs). To address this issue we used two different methods of EEG analysis: standardized low-resolution electromagnetic tomography (sLORETA) and normative independent component analysis (NICA) recently described by Congedo et al. (2010). sLORETA (Pascual-Marqui, 2002) is a widely used inverse solution technique that estimates the intracranial distribution of electrical activity in the cortex based on a head model. ICA is a data-driven (i.e. model-free) technique widely used to decompose the multivariate EEG signal into sources as independent as possible (Congedo et al., 2008; Onton et al., 2006). The assumption of EEG source independence is consistent with the fact that the cortex is organized into functionally distinct areas and that neighbouring and highly connected regions (e.g. via corpus callosum) are likely to fire in synchrony (Onton et al., 2006). Physical and statistical principles supporting the use of decomposition methods based on secondorder statistics for EEG data have been reviewed in Congedo et al. (2008).

Whereas SLORETA focuses on voxel by voxel analysis without searching for relationships between them, ICA separates the signal based on its intrinsic relationships. For example, sLORETA may identify abnormal power in a cluster containing a number of independent sources that ICA would split into several components. On the other hand ICA would group correlated sources into the same components, even if they are distant in space from each other. The two methods provide complementary information and their application on the same data may provide a more comprehensive and consistent view, especially considering a high sample size as in this study.

\section{METHODS}

\section{Subjects}

Fifty in-patients diagnosed with OCD according to ICD-10 (World Health Organisation, 2009) and DSM-IV (American Psychiatric Association, 1994) criteria and 50 healthy controls matched for age, sex and handedness were included in the study (Table 1). Exclusion criteria involved concurrent severe or chronic medical disease, substance abuse, mental retardation, organic mental disorder, lifetime history of psychosis, mood disorders, severe head injury and neurosurgery. In addition, controls were required to have no history of any mental disorder. At the time of EEG recording, 20 patients were drug-free and 30 were using SSRIs medication. The medication status was stable for at least four weeks prior to the study. Besides the diagnosis of OCD, the rationale to include both SSRIs medicated and drug-free patients was the presence of marked clinical symptoms that could possibly be reflected in EEG. At the same time, the drug-free subgroup served as a reference group allowing a control of the effect of the SSRIs medication. Clinical data in the patient group included age of OCD onset, illness duration and symptom severity (Table 1). Symptom severity was assessed using the Yale-Brown Obsessive Compulsive Scale (Y-BOCS) (Goodman et al., 1989). The level of general anxiety was measured with the Hamilton Anxiety Rating Scale (HAMA) (Hamilton, 1959). The study was carried out in accordance with the Declaration of Helsinki and written informed consent was obtained from all subjects.

\section{EEG recording and data preprocessing}

EEG was recorded during eyes-closed resting state on a BrainScope differential amplifier (Unimedis, Ltd., Czech Republic) against the AFz, FCz or Cz reference. The signal was obtained from 19 scalp locations according to the international 10-20 system using an ECl electro-cap (Electro-Cap International, Inc., Eaton, USA). The EEG recording system acquired the data with a 16 -bit depth and a $7.63 \mathrm{nV} / \mathrm{bit}$ resolution (i.e. approx. $130 \mathrm{bit} / \mu \mathrm{V}$ ) and a dynamic range of $\pm 250 \mu \mathrm{V}$. The data were sampled at a rate of 250 or $256 \mathrm{~Hz}$ and filtered with a $0.15-$ $70 \mathrm{~Hz}$ band-pass filter. 
Data were imported to EureKa software (NovaTechEEG, Inc., Mesa, Arizona, USA) to remove episodic artefacts. If necessary, continuous muscle artefacts were removed as independent components using ICoN software (http://sites.google.com/site/marcocongedo/software/nica). This procedure was done by a trained clinician with a special attention to K-complexes and was equal in both groups. At least $60 \mathrm{~s}$ of artefact-free data were analyzed in subsequent analysis. Before analysis, all data filtered between $2-45 \mathrm{~Hz}$ were re-referenced against the average reference montage and re-sampled at $128 \mathrm{~Hz}$. Several comparisons of subgroups recorded with different references and re-referenced against the average reference montage were performed by means of sLORETA and normative group ICA confirming that there was no systematic effect due to different recording references.

Mean normalized absolute power at all electrodes for both groups of subjects is shown in Figure 1.

\section{sLORETA analysis}

Average Fourier cross-spectral matrices were computed for each frequency in the range of $2-45 \mathrm{~Hz}$ with $1 \mathrm{~Hz}$ resolution as well as for 11 frequency bands defined analogous to the previous LORETA study in obsessivecompulsive subjects (Sherlin and Congedo, 2005): delta $(2-3.5 \mathrm{~Hz})$, theta $(4-7.5 \mathrm{~Hz})$, alpha1 $(8-10 \mathrm{~Hz})$, alpha2 $(10-12 \mathrm{~Hz})$, beta1 $(12-16 \mathrm{~Hz})$, beta2 $(16-20 \mathrm{~Hz})$, beta3 $(20-24 \mathrm{~Hz})$, beta4 $(24-28 \mathrm{~Hz})$, beta5 (28 $32 \mathrm{~Hz})$, gamma $(32-45 \mathrm{~Hz})$ and total band $(2-45 \mathrm{~Hz})$. Subsequently, absolute and relative current density power were computed in 2394 cortical voxels using sLORETA and the three-shell spherical head model coregistered with the Talairach atlas as implemented in the LORETA-Key software (Key Institute for Brain-Mind Research, Zurich, Switzerland). Current density power was normalized in the solution volume. Absolute and relative current density data were log transformed, smoothed with a $14 \mathrm{~mm}$ spatial moving average filter and compared between groups (drug-free vs. medicated patients, all patients vs. controls, drug-free patients vs. controls and medicated patients vs. controls) by means of randomization-permutation statistics (100 000 permutations) using MHyT software (NovaTechEEG, Inc.). All bands were treated simultaneously in the $t$ between two-sided max-statistics test guaranteeing that the family-wise type I error (FWE) did not exceed the nominal level (0.05). Mean current densities in the significant regions of interest (ROIs) were extracted and a correlation analysis was performed to investigate their relationships with clinical symptoms as assessed with $\mathrm{Y}$ BOCS, the level of general anxiety measured with HAMA, age of onset and illness duration. A partial correlation analysis was used to eliminate the potential confounding effect of age. Before the analysis, all data were tested for severe departures from gaussianity.

\section{Group ICA and group comparison}

We employed the blind source separation approach described in Congedo et al. (2008) that can separate uncorrelated sources provided they have non-proportional power spectrum. For normal brain we have reasons to suppose that a great majority of healthy individuals share the same resting-state components (discussed in Congedo et al., 2010) which ensures that the group components are meaningful. However, the same may not be true for the clinical sample, which is in general much more heterogeneous in terms of EEG features. Therefore, in this research, we have focused on the identification of normal EEG features that deviate significantly in the OCD population.

The procedure used in this study is fully explained in Congedo et al. (2010). In brief, we estimated Fourier cospectral matrices for each subject in the control group and each frequency. Group ICA was performed as the approximate joint diagonalization of the grand-average Fourier cospectral matrices. We diagonalized cospectra in the range of $2-26 \mathrm{~Hz}$. Based on the previous study by Congedo et al. (2010) on large samples of normal individuals we extracted the seven most energetic components (normative components). The resulting demixing matrix was used to extract the power of the same components from the OCD group as well as to compute the mixing matrix which contains in columns scalp spatial pattern associated with each components and thus can serve as an input for SLORETA to estimate their cortical location. For each component, absolute, relative and normal power (normal power indicates for each frequency the proportion of power expressed by each component compared to the all of them) were computed with $1 \mathrm{~Hz}$ resolution and compared to normative data obtained from the control group using the randomization-permutation multiple-comparison approach as in SLORETA. The individual power values were used for correlation analyses as described above.

\section{RESULTS}

\section{Demographic data}

All groups were equivalent for age, sex and handedness. Drug-free and SSRIs medicated OCD patients did not differ in clinical characteristics with the exception of higher compulsion score in medicated patients (Table 1). The mean Y-BOCS scores 19.4 indicates marked severity of obsessive-compulsive symptoms. 


\section{sLORETA}

Drug-free and SSRIs medicated patients did not differ from each other in absolute or in relative power, therefore they were further tested as a unitary group. Compared with controls, OCD patients had higher normalized absolute current density power at low frequencies $(2-6 \mathrm{~Hz})$ in the medial frontal cortex (anterior cingulate, medial frontal gyrus, medial orbitofrontal cortex) and at 2 and $3 \mathrm{~Hz}$ frequencies in the left parahippocampal gyrus and uncus (Table 2). The highest $t$-values were localized in the dorsal anterior cingulate $\left(t_{\max }=4.24, p<0.05\right.$, FWE corrected, Figure 2$)$. A comparison of the SSRIs and control groups yielded the same results $\left(t_{\max }=4.39, p<0.05\right.$, FWE corrected). A comparison of drug-free patients and controls was not significant at $p<0.05$, however, moving the $t$-value threshold down the same pattern of increased low frequencies in the medial frontal cortex was seen also in drug-free OCD patients (local $t$-value maxima 2.44, $2.58,3.07,3.32,3.33$ were found at 2, 3, 4, 5 and $6 \mathrm{~Hz}$ respectively, Figure 3). In a broader band comparison, delta and theta absolute current density power were higher in $\operatorname{OCD}\left(t_{\max }=4.39, p<0.05\right.$, FWE corrected).

Relative power also tended to be higher in OCD patients in the same region and frequencies as absolute power, however, the significance threshold was exceeded only at $5 \mathrm{~Hz}$ and was localized in the dorsal ACC. Additionally, an increase in relative current density at 18 and $19 \mathrm{~Hz}$ was found in a very small cluster ( 7 and 3 voxels, respectively) in the right superior and middle temporal gyrus ( $t_{\max }=3.77, p<0.05$, FWE corrected). In broader band testing, OCD patients had higher relative power in the beta2 band in a small area of the right superior and middle temporal gyrus $\left(t_{\max }=3.57, p<0.05\right.$, FWE corrected).

Correlation analysis did not reveal any relationship between low-frequency current density power and obsessive-compulsive symptoms, anxiety, age of onset and illness duration.

\section{Group ICA}

The 7 components separated through the group independent component analysis performed on the whole control sample are shown in Figure 4. Only component 5 differed in power between OCD patients and controls $(p<0.05$, corrected). Relative power was higher in OCD at low frequencies $(3-6 \mathrm{~Hz})$ and normal power was elevated at $2-6 \mathrm{~Hz}$ and at $20 \mathrm{~Hz}$ (Figure 5). The local maxima of this component were localized in subgenual anterior cingulate (BA 25), subcallosal gyrus (BA 34), rectal gyrus (BA 11), insula (BA 13), superior temporal and parahippocampal gyrus (BA 38), midcingulate (BA 24) and marginally in lateral frontal lobe (BA 6, 8, 9). The component was bilateral and more pronounced on the left side. When comparing separately, neither drug-free nor medicated patients showed any difference compared with controls at the adopted threshold $(p<0.05$, corrected). However, both groups displayed the same pattern of difference from controls at lower significance level $(p<0.1$, corrected).

Correlation analysis did not reveal any relationship between component 5 power at frequencies that were significant in the inter-group analysis and obsessive-compulsive (Y-BOCS) or anxiety (HAMA) rating scale scores. Age of onset showed a trend towards a positive correlation with normal power in component 5 at 4 and $5 \mathrm{~Hz}(r$ $=0.27, p=0.078$, resp. $r=0.27, p=0.082$ ).

\section{DISCUSSION}

Both methods, sLORETA and group ICA, indicated abnormal functioning of medial frontal cortex in OCD. The results converge with the leading orbitofronto-striatal model of $O C D$ as well as with the performance monitoring hypothesis.

The orbitofronto-striatal model postulating hyperactivation of the medial frontal cortex has been reported using a variety of methodological approaches (Rotge et al., 2008; Yucel et al., 2007). Low frequencies generated in the frontal midline cortex have been connected with mental activity (Onton et al., 2005) and higher cerebral glucose metabolism (Leuchter et al., 1999; Pizzagalli et al., 2003). Thus, our findings are in agreement with medial frontal hyperactivation in OCD. In line with performance monitoring hypothesis and ERN findings in OCD, sLORETA revealed an excess in absolute power at slow frequencies peaking at 4 and $5 \mathrm{~Hz}$ and reaching maximal $t$-values in dorsal ACC. Studies show that ERN arises at least in part from ongoing theta activity of $4-7$ $\mathrm{Hz}$ (Luu et al., 2003; Luu et al., 2004; Luu and Tucker, 2001; Trujillo and Allen, 2007). Although the main role in performance monitoring has been attributed to dorsal medial frontal cortex, it has been shown that other medial frontal and limbic structures (Luu et al., 2003) are necessary for the generation of ERN. Accordingly, the low-frequency excess revealed by SLORETA in our study was not confined to dorsal ACC but included the entire ACC, medial orbitofrontal cortex and part of the left parahippocampal gyrus.

The independent component that differentiated patients and controls received the strongest signal from the subgenual part of anterior cingulate. This structure is an important gateway in the regulation of neural circuits involved in mood and anxiety disorders (Van et al., 2006). It has been shown that metabolic activity in subgenual ACC predicts therapeutic response to anterior capsular electrostimulation in patients with OCD and 
comorbid depression and decreases post-treatment (Van et al., 2006). Interestingly, the most promising targets of deep brain stimulation in OCD and depression focus on fronto-striatal pathways, especially on fibres connecting the subgenual anterior cingulate and orbital cortex with basal ganglia, thalamus, and amygdala (Haber and Brucker, 2009).

In the context of the enhancing effect of negative affect on ERN (Luu et al., 2000), we hypothesize that the hyperactive neural circuit of subgenual ACC represented by the component which is abnormal in OCD might impact on possibly vulnerable dorsal ACC and thus lead to generation of obsessive-compulsive symptoms as suggested by Pitman (1987).

The component that was abnormal in OCD in our study was localized more on the left side and included the insula, parahippocampal gyrus, midcingulate and lateral frontal cortex. The increasing body of evidence indicates that lateral frontal cortex is necessary for the performance monitoring system (Maltby et al., 2005; Ridderinkhof et al., 2004) and there is also support for the insular contribution in error detection (Menon et al., 2001). Moreover, insula is connected with ACC and midcingulate (Taylor et al., 2009), other parts of the neural circuit represented by the component. It has been suggested that the performance monitoring network communicates via transient synchronous oscillations in the theta range (Cavanagh et al., 2009). Interestingly, already Gevins et al. (1989) revealed that theta activity over the frontal midline sites was related to theta recorded at the left lateral frontal electrodes during error feedback signal. Our findings involve the classic theta $(4-8 \mathrm{~Hz})$ as well as delta bands encompassing even lower frequencies. However, given that the abnormalities in both bands concerned the same location and component, we suggest that the low frequency increases at $2-$ $6 \mathrm{~Hz}$ reflect the same pathophysiological process. Interestingly, a recent factor analysis indicated similarities between low frequencies and proposed a broader low frequency band ranging from $1.5 \mathrm{~Hz}$ to $7 \mathrm{~Hz}$ (PascualMarqui et al., 2007). Moreover, it has been found that at low frequencies, the highest correlation with cerebral perfusion as measured by positron emission tomography was in the $2-6 \mathrm{~Hz}$ frequency band (Leuchter et al., 1999) which corresponds with our findings. Although most ERP studies used a $4 \mathrm{~Hz}$ high-pass filter and thus $a$ priori excluded lower frequencies (Luu et al., 2003; Luu et al., 2004; Luu and Tucker, 2001), delta activity has also been related to performance monitoring processes (Yordanova et al., 2004).

Another approach that might explain our findings is based on the concept of thalamocortical dysrhythmia (Llinás et al., 1999). Thalamocortical dysrhythmia is a disrupted dynamic between thalamus and cortex arising due to changes in the behavior of thalamic cells caused by their hyperpolarization. This leads to pathological theta oscialtion in the cortex. In neuropsychiatric symptoms the areas where we expect to see maximal lowfrequency activity include the cingulate, medial prefrontal, and orbitofrontal cortices (Llinás et al., 1999) which corresponds with our findings.

Our results indicate that medial frontal low-frequency excess was present in both the drug-free and SSRIs medicated group and in SLORETA it was even more pronounced in the SSRIs group. This suggests that the increased low-frequency power was not due to medication and, moreover, that SSRIs did not normalize this EEG pattern. Previous studies in OCD show that treatment response to serotonin reuptake inhibitors is linked with a decrease in alpha power which is enhanced pre-treatment in responding patients (Hansen et al., 2003; Prichep et al., 1993). On the other hand, pre-treatment EEG analysis in patients who did not respond to serotonin reuptake inhibitors showed an excess in the theta frequency band (Prichep et al., 1993). On average, our patients had marked obsessive-compulsive symptoms and had been suffering from OCD for more than twelve years despite previous treatment. Therefore, it is plausible that our sample included a high percentage of SSRIs non-responders. In our opinion, this is the main reason why our results differ from those reported by Velikova et al. (2010). Although in the context of the electrophysiological effect of serotonin reuptake inhibitors in OCD and the presence of low-frequency excess in both medicated and drug-free patients ( $a$ trend in SLORETA and a significant finding in group ICA analysis) it does not seem likely that the results would be due to poor treatment adherence in medicated patients, it should be acknowledged that EEG was recorded at the beginning of hospitalization and that we do not have data about prior treatment adherence. An interesting view of these results would be provided by extending the study to clinical control groups that would allow us to resolve the question of whether our findings are specific for OCD or whether they represent a more general characteristic of a broader diagnostic category.

The fact that low-frequency power excess did not correlate with clinical data may have several reasons. Firstly, clinical rating scales evaluate symptom severity experienced up to one week before, whereas EEG recording comprises only a few minutes. Secondly, inter-individual differences in expectations and anxiety during EEG examination might have confounded the results. Finally, similarly to anatomical and functional abnormalities (Chamberlain et al., 2008; Menzies et al., 2007) our findings may represent a neurobiological substrate for the disorder rather than a correlate of symptom severity. 
Several limitations of this study should be mentioned. First, the identified regional activity cannot be assumed to measure neuronal activity at those sites. It is instead used to infer an activity that is consistent with the sLORETA model. Source localization is susceptible to errors and it is less accurate than direct measurements from anatomical regions. The spatial resolution of the sLORETA method is low and a confounding effect comes also from the use of a standard head model that was applied in our study as individual MRI anatomical data were not available.

Second, the seven separated components were only partially identical with those reported in Congedo et al. (2010). The components 1, 3 and 5 in our sample correspond with those described in Congedo et al. (2010) in terms of both, spatial and spectral distribution. The component 4 has a similar spatial distribution as the corresponding component in Congedo et al. (2010), however it does not have low normal alpha power. On the other hand, the components 2 and 6 correspond with their counterparts in the power distribution but their localization is only partially identical. We did not find the anterior cingulate component (component 5 in Congedo et al., 2010) and found instead an extra component localized in BA 6 and the adjacent regions (component 7). There are several reasons for this discrepancy. First, the mean age of our sample was higher than the age of subjects in both databases used in Congedo et al. (in 2010). Second, the sample size in all databases might not be high enough. Third, our data were re-referenced to average reference montage before the analysis which we believe to be the major factor explaining the divergence of the result. However, despite the different settings and machines used in the three samples, the overall similarities are substantial.

We are aware of a special attention that should be paid to ocular artifacts because the main result of our study is at low frequencies. Although we did not record electrooculogram, we are confident that the main result is not due to eye movement artifacts because 1 ) the samples are large, 2) the peak effect was observed at 4 and $5 \mathrm{~Hz}$, whereas the effect due to eye movements would peak at very low frequencies, 3) there were visible lowfrequency abnormalities in the raw EEG in the OCD group described by a certified neurologist as focal $4-5 \mathrm{~Hz}$ theta with highest amplitude at electrodes F7, Fz, F3 or T3 without simultaneous amplitude increase (or any sign of propagation) at Fp1 or Fp2. Another potential confounding factor at low frequencies may be the presence of K-complexes. Although the artefact-free segments in our study were selected with a special attention to K-complexes, we asked a certified neurologist to independently review the data. He confirmed that OCD subjects did not present enhanced K-complexes during resting state EEG and, therefore, that the results are not due to K-complexes.

A long research tradition has shown the importance to consider individually-defined frequency bands for EEG analysis. For example, Klimesch and colleagues described three narrow alpha sub-bands that can be defined in each individual based on the alpha peak frequency and showed their different physiological meaning (Klimesch, 1999; Klimesch et al., 1997). According to Klimesch (1999) the individual alpha frequency should be used as a reference point not only for adjusting different alpha frequency sub-bands but also for theta range definition (Klimesch, 1999). Because our results are also within the theta band, we tested if there are differences in the alpha peak between OCD patients and healthy controls. We extracted the alpha peak frequency with $0.5 \mathrm{~Hz}$ resolution for each individual and compared the groups. The mean alpha peak in the patient group was $10.54 \pm 1.059 \mathrm{~Hz}$ and the mean alpha peak in the control group was $10.36 \pm 0.969 \mathrm{~Hz}$. The groups did not differ in the alpha peak frequency $(p=0.370)$. Therefore, on these samples it is not necessary to individualize the band definition.

In summary, our study presents the largest set of results concerning resting state EEG in OCD patients. Consistently with previous EEG studies it confirms a differential EEG pattern between OCD patients and healthy controls. Although this study was not conceived to distinguish between SSRIs responders and non-responders, it supports previous findings on theta excess in non-responding patients. In addition, it provides information about the sources of enhanced low-frequency activity in OCD. Their localization in the medial frontal cortex and mainly in ACC is congruent with neuroimaging findings on medial frontal hyperactivation in OCD as well as with the performance monitoring hypothesis. Both the sLORETA and group ICA methods deserve further investigation as diagnostic tools. Our results are of practical interest for potential nonpharmacological treatment such a neurofeedback intervention aimed at normalizing EEG patterns.

\section{References:}

American Psychiatric Association. Diagnostic and statistical manual of mental disorders (DSM-IV), 4th ed. Washington DC: American Psychiatric Association, 1994. 
Bolwig TG, Hansen ES, Hansen A, Merkin H, Prichep LS. Toward a better understanding of the pathophysiology of OCD SSRI responders: QEEG source localization. Acta Psychiatr Scand 2007;115:237-242.

Cavanagh JF, Cohen MX, Allen JJ. Prelude to and resolution of an error: EEG phase synchrony reveals cognitive control dynamics during action monitoring. J Neurosci 2009;29:98-105.

Chamberlain SR, Menzies L, Hampshire A, Suckling J, Fineberg NA, del Campo N, Aitken M, Craig K, Owen AM, Bullmore ET, Robbins TW, Sahakian BJ. Orbitofrontal dysfunction in patients with obsessive-compulsive disorder and their unaffected relatives. Science 2008;321:421-422.

Congedo M, Gouy-Pailler C, Jutten C. On the blind source separation of human electroencephalogram by approximate joint diagonalization of second order statistics. Clin Neurophysiol 2008;119:2677-2686.

Congedo M, John ER, De Ridder D, Prichep L. Group Independent Component Analysis of Resting-State EEG in Large Normative Samples. Int J of Psychophysiol 2010; ;78:89-99.

Debener S, Ullsperger M, Siegel M, Fiehler K, von Cramon DY, Engel AK. Trial-by-trial coupling of concurrent electroencephalogram and functional magnetic resonance imaging identifies the dynamics of performance monitoring. J Neurosci 2005;25:11730-11737.

Dehaene S, Posner MI, Tucker DM. Localization of a neural system for error detection and compensation. Psychol Sci 1994;5:303-305.

Endrass T, Klawohn J, Schuster F, Kathmann N. Overactive performance monitoring in obsessive-compulsive disorder: ERP evidence from correct and erroneous reactions. Neuropsychologia 2008;46:1877-1887.

Fitzgerald KD, Welsh RC, Gehring WJ, Abelson JL, Himle JA, Liberzon I, Taylor SF. Error-related hyperactivity of the anterior cingulate cortex in obsessive-compulsive disorder. Biol Psychiatry 2005;57:287-294

Fontenelle LF, Mendlowicz MV, Ribeiro P, Piedade RA, Versiani M. Low-resolution electromagnetic tomography and treatment response in obsessive-compulsive disorder. Int J Neuropsychopharmacol 2006;9:89-94.

Gevins AS, Cutillo BA, Bressler SL, Morgan NH, White RM, Illes J, Greer DS. Event-related covariances during a bimanual visuomotor task. II. Preparation and feedback. Electroencephalogr Clin Neurophysiol 1989;74:147-160.

Goodman WK, Price LH, Rasmussen SA, Mazure C, Fleischmann RL, Hill CL, Heninger GR, Charney DS. The Yale-Brown Obsessive Compulsive Scale. I. Development, use, and reliability. Arch Gen Psychiatry 1989;46:1006-1011.

Gu BM, Park JY, Kang DH, Lee SJ, Yoo SY, Jo HJ, Choi CH, Lee JM, Kwon JS. Neural correlates of cognitive inflexibility during taskswitching in obsessive-compulsive disorder. Brain 2008;131:155-164.

Haber SN, Brucker JL. Cognitive and limbic circuits that are affected by deep brain stimulation. Front Biosci 2009;14:1823-1834.

Hansen ES, Prichep LS, Bolwig TG, John ER. Quantitative electroencephalography in OCD patients treated with paroxetine. Clin Electroencephalogr 2003;34:70-74

Hamilton M. The assessment of anxiety states by rating. Br J Med.Psychol 1959;32:50-55.

Karadag F, Oguzhanoglu NK, Kurt T, Oguzhanoglu A, Atesci F, Ozdel O. Quantitative EEG analysis in obsessive compulsive disorder. Int J Neurosci 2003;113:833-847.

Karno M, Golding JM, Sorenson SB, Burnam MA. The epidemiology of obsessive-compulsive disorder in five US communities. Arch Gen Psychiatry 1988;45:1094-1099.

Klimesch W. EEG alpha and theta oscillations reflect cognitive and memory performance: a review and analysis. Brain Res Brain Res Rev 1999;29:169-195

Klimesch W, Doppelmayr M, Pachinger T, Ripper B. Brain oscillations and human memory performance: EEG correlates in the upper alpha and theta bands. Neurosci Lett 1997;238:9-12

Leuchter AF, Uijtdehaage SH, Cook IA, O'Hara R, Mandelkern M. Relationship between brain electrical activity and cortical perfusion in normal subjects. Psychiatry Res 1999;90:125-140.

Llinás RR, Ribary U, Jeanmonod D, Kronberg E, Mitra PP. Thalamocortical dysrhythmia: A neurological and neuropsychiatric syndrome characterized by magnetoencephalography. Proc Natl Acad Sci U S A 1999;96:15222-15227.

Luu P, Flaisch T, Tucker DM. Medial frontal cortex in action monitoring. J Neurosci 2000;20:464-469.

Luu P, Tucker DM. Regulating action: alternating activation of midline frontal and motor cortical networks. Clin Neurophysiol 2001;112:1295-1306. 
Luu P, Tucker DM, Derryberry D, Reed M, Poulsen C. Electrophysiological responses to errors and feedback in the process of action regulation. Psychol Sci 2003;14:47-53.

Luu P, Tucker DM, Makeig S. Frontal midline theta and the error-related negativity: neurophysiological mechanisms of action regulation. Clin Neurophysiol 2004;115:1821-1835.

Maltby N, Tolin DF, Worhunsky P, O'Keefe TM, Kiehl KA. Dysfunctional action monitoring hyperactivates frontal-striatal circuits in obsessive-compulsive disorder: an event-related fMRI study. Neuroimage 2005;24:495-503.

Menon V, Adleman NE, White CD, Glover GH, Reiss AL. Error-related brain activation during a Go/NoGo response inhibition task. Hum Brain Mapp 2001;12:131-143.

Menzies L, Achard S, Chamberlain SR, Fineberg N, Chen CH, del Campo N, Sahakian BJ, Robbins TW, Bullmore E. Neurocognitive endophenotypes of obsessive-compulsive disorder. Brain 2007;130:3223-3236.

Menzies L, Chamberlain SR, Laird AR, Thelen SM, Sahakian BJ, Bullmore, ET. Integrating evidence from neuroimaging and neuropsychological studies of obsessive-compulsive disorder: the orbitofronto-striatal model revisited. Neurosci Biobehav Rev 2008a;32:525-549.

Menzies L, Williams GB, Chamberlain SR, Ooi C, Fineberg N, Suckling J, Sahakian BJ, Robbins TW, Bullmore ET. White matter abnormalities in patients with obsessive-compulsive disorder and their first-degree relatives. Am J Psychiatry 2008b;165:1308-1315.

Nabeyama M, Nakagawa A, Yoshiura T, Nakao T, Nakatani E, Togao O, Yoshizato C, Yoshioka K, Tomita M, Kanba S. Functional MRI study of brain activation alterations in patients with obsessive-compulsive disorder after symptom improvement. Psychiatry Res 2008;163:236-247

Onton J, Delorme A, Makeig S. Frontal midline EEG dynamics during working memory. Neuroimage 2005;27:341-356.

Onton J, Westerfield M, Townsend J, Makeig S. Imaging human EEG dynamics using independent component analysis. Neurosci Biobehav Rev 2006;30:808-822.

Pascual-Marqui RD. Standardized low-resolution brain electromagnetic tomography (sLORETA): technical details. Methods Find Exp Clin Pharmacol 2002;24 Suppl D:5-12.

Pascual-Marqui RD, Lehmann D, Esslen M, Kochi K, Koukkou M. sLORETA-based functional segmentation of the human cortex during REST. Applied Neuroscience for Healthy Brain Function 2007, the 17-20 ${ }^{\text {th }}$, Nijmegen. Retrieved from http://www.appliedneuroscience.nl/file.php?fId=149.

Pitman RK. A cybernetic model of obsessive-compulsive psychopathology. Compr Psychiatry 1987;28:334-343.

Pizzagalli DA, Oakes TR, Davidson RJ. Coupling of theta activity and glucose metabolism in the human rostral anterior cingulate cortex: an EEG/PET study of normal and depressed subjects. Psychophysiology 2003;40:939-949.

Pogarell O, Juckel G, Mavrogiorgou P, Mulert C, Folkerts M, Hauke W, Zaudig M, Moller HJ, Hegerl U. Symptom-specific EEG power correlations in patients with obsessive-compulsive disorder. Int J Psychophysiol 2006;62:87-92.

Prichep LS, Mas F, Hollander E, Liebowitz M, John ER, Almas M, DeCaria CM, Levine RH. Quantitative electroencephalographic subtyping of obsessive-compulsive disorder. Psychiatry Res 1993;50:25-32.

Remijnse PL, Nielen MM, van Balkom AJ, Cath DC, van Open P, Uylings HB, Veltman DJ. Reduced orbitofrontal-striatal activity on a reversal learning task in obsessive-compulsive disorder. Arch Gen Psychiatry2006;63:1225-1236.

Ridderinkhof KR, Ullsperger M, Crone EA, Nieuwenhuis S. The role of the medial frontal cortex in cognitive control. Science 2004;306:443-447.

Rotge JY, Guehl D, Dilharreguy B, Cuny E, Tignol J, Bioulac B, Allard M, Burbaud P, Aouizerate B. Provocation of obsessive-compulsive symptoms: a quantitative voxel-based meta-analysis of functional neuroimaging studies. J Psychiatry Neurosci 2008;33:405-412.

Roth RM, Saykin AJ, Flashman LA, Pixley HS, West JD, Mamourian AC. Event-related functional magnetic resonance imaging of response inhibition in obsessive-compulsive disorder. Biol Psychiatry 2007;62:901-909.

Sherlin L, Congedo M. Obsessive-compulsive dimension localized using low-resolution brain electromagnetic tomography (LORETA) Neurosci Lett 2005;387:72-74.

Taylor KS, Seminowicz DA, Davis KD. Two systems of resting state connectivity between the insula and cingulate cortex. Hum Brain Mapp $2009 ; 30: 2731-2745$

Trujillo LT, Allen JJ. Theta EEG dynamics of the error-related negativity. Clin Neurophysiol 2007;118:645-668. 
Ullsperger M, von Cramon DY. The role of intact frontostriatal circuits in error processing. J Cogn Neurosci 2006;18:651-664.

Ursu S, Stenger VA, Shear MK, Jones MR, Carter CS. Overactive action monitoring in obsessive-compulsive disorder: evidence from functional magnetic resonance imaging. Psychol Sci 2003;14:347-353.

van den Heuvel OA, Remijnse PL, Mataix-Cols D, Vrenken H, Groenewegen HJ, Uylings HB, van Balkom AJ, Veltman DJ. The major symptom dimensions of obsessive-compulsive disorder are mediated by partially distinct neural systems. Brain 2009;132:853-868.

van den Heuvel OA, Veltman DJ, Groenewegen HJ, Cath DC, van Balkom AJ, van Hartskamp J, Barkhof F, van Dyck R. Frontal-striatal dysfunction during planning in obsessive-compulsive disorder. Arch Gen Psychiatry 2005;62:301-309.

Van Laere K, Nuttin B, Gabriels L, Dupont P, Rasmussen S, Greenberg BD, Cosyns P. Metabolic imaging of anterior capsular stimulation in refractory obsessive-compulsive disorder: a key role for the subgenual anterior cingulate and ventral striatum. J Nucl Med 2006;47:740-747.

Velikova S, Locatelli M, Insacco C, Semeraldi E, Comi G, Leocani L. Dysfunctional brain circuitry in obsessive-compulsive disorder: source and coherence analysis of EEG rhythms. Neuroimage 2010;49:977-983.

World Health Organisation (WHO). ICD-10: The ICD-10 classification of mental and behavioural disorders: clinical descriptions and diagnostic guidelines. Geneva: World Health Organisation, 1992.

Yordanova J, Falkenstein M, Hohnsbein J, Kolev V. Parallel systems of error processing in the brain. Neuroimage 2004;22:590-602.

Yücel M, Harrison BJ, Wood SJ, Fornito A, Wellard RM, Pujol J, Clarke K, Phillips ML, Kyrios M, Velakoulis D, Pantelis C. Functional and biochemical alterations of the medial frontal cortex in obsessive-compulsive disorder. Arch Gen Psychiatry 2007;64:946-955. 
Tables:

Table 1: Demographic and clinical characteristics of the groups of subjects.

\begin{tabular}{|c|c|c|c|c|c|c|c|c|c|c|c|c|c|}
\hline \multirow[b]{2}{*}{ Sample characteristics } & \multicolumn{2}{|c|}{$\begin{array}{c}\text { OCD } \\
(N=50)\end{array}$} & \multicolumn{2}{|c|}{$\begin{array}{l}\text { controls } \\
(N=50)\end{array}$} & \multicolumn{2}{|c|}{$\begin{array}{l}\text { OCD - DF } \\
(N=20)\end{array}$} & \multicolumn{2}{|c|}{$\begin{array}{c}\text { OCD - SSRIS } \\
(\mathrm{N}=30)\end{array}$} & \multirow[b]{2}{*}{ stat. test } & \multirow{2}{*}{$\begin{array}{c}\begin{array}{c}\text { OCD vs. } \\
\text { controls }\end{array} \\
p \\
\end{array}$} & \multirow{2}{*}{$\begin{array}{c}\text { OCD - DF } \\
\text { vs. controls } \\
p\end{array}$} & \multirow{2}{*}{$\begin{array}{l}\text { OCD - SSRIs } \\
\text { vs. controls } \\
p\end{array}$} & \multirow{2}{*}{$\begin{array}{c}\text { OCD-DF vs } \\
\text { OCD-SSRIs } \\
\boldsymbol{p}\end{array}$} \\
\hline & mean & SD & mean & SD & mean & SD & mean & SD & & & & & \\
\hline \multicolumn{14}{|l|}{ Demographic } \\
\hline age (years) & 29.2 & 5.0 & 28.4 & 5.6 & 28.4 & 4.7 & 29.1 & 5.3 & Wilcoxon & 0.319 & 0.303 & 0.533 & 0.781 \\
\hline sex (men:women) & $20: 30$ & NA & $23: 27$ & NA & $7: 13$ & NA & $13: 17$ & NA & chi-square & 0.545 & 0.401 & 0.816 & 0.556 \\
\hline handedness (right:left) & $48: 2$ & NA & $48: 2$ & NA & 19:1 & NA & $29: 1$ & NA & chi-square & 1.000 & 0.852 & 0.879 & 0.768 \\
\hline \multicolumn{14}{|l|}{ Clinical } \\
\hline obsessions & 9.8 & 3.4 & NA & NA & 9.4 & 3.6 & 10.0 & 3.3 & $t$-test & NA & NA & NA & 0.576 \\
\hline compulsions & 9.8 & 5.0 & NA & NA & 7.5 & 4.4 & 11.0 & 5.0 & $t$-test & NA & NA & NA & 0.040 \\
\hline $\operatorname{HAMA}(n=35)$ & 12.7 & 6.0 & NA & NA & 12.7 & 5.0 & 12.7 & 6.5 & $t$-test & NA & NA & NA & 0.979 \\
\hline age of onset (years) & 16.9 & 7.2 & NA & NA & 18.1 & 6.9 & 16.1 & 7.4 & $t$-test & NA & NA & NA & 0.406 \\
\hline illness duration (years) & 12.5 & 7.9 & NA & NA & 11.2 & 8.8 & 13.3 & 7.4 & $t$-test & NA & NA & NA & 0.402 \\
\hline
\end{tabular}

Abbreviations: Y-BOCS - Yale-Brown Obsessive-Compulsive scale, SD - standard deviation, DF - drug-free, SSRIs - selective serotonin reuptake inhibitors, NA - not applied/not applicable

Table 2: Number and localization of voxels with excessive absolute current density in OCD compared with controls $(p<0.05)$. The structures are reported in their mean $t$-value order.

\begin{tabular}{|c|c|c|c|c|c|c|c|c|c|c|}
\hline \multirow[b]{2}{*}{ Brain structure and Brodmann area } & \multicolumn{2}{|c|}{$2 \mathrm{~Hz}$} & \multicolumn{2}{|c|}{$3 \mathrm{~Hz}$} & \multicolumn{2}{|c|}{$4 \mathrm{~Hz}$} & \multicolumn{2}{|c|}{$5 \mathrm{~Hz}$} & \multicolumn{2}{|c|}{$6 \mathrm{~Hz}$} \\
\hline & mean $t$ & voxels & mean $t$ & voxels & mean $t$ & voxels & mean $t$ & voxels & mean $t$ & voxels \\
\hline Anterior cingulate (BA 24, 25, 32, 33) & 4.86 & 45 & 4.91 & 49 & 5.26 & 55 & 5.26 & 58 & 5.04 & 55 \\
\hline Cingulate gyrus (BA 24, 32) & 4.65 & 21 & 4.76 & 27 & 4.82 & 38 & 4.83 & 31 & 4.60 & 17 \\
\hline Subcallosal gyrus (BA 25, 34) & 4.70 & 15 & 4.65 & 14 & 4.77 & 14 & 4.78 & 10 & 4.62 & 8 \\
\hline Medial frontal gyrus (BA 9, 10, 11, 25) & 4.58 & 22 & 4.55 & 29 & 4.71 & 45 & 4.79 & 51 & 4.62 & 47 \\
\hline Rectal gyrus (BA 11) & 4.57 & 14 & 4.59 & 14 & 4.62 & 15 & 4.57 & 17 & 4.40 & 12 \\
\hline Left parahippocampal gyrus (BA $28,34,35,37$ ) & 4.48 & 16 & 4.39 & 9 & 4.42 & 5 & 4.71 & 1 & n.s. & 0 \\
\hline Inferior frontal gyrus (BA 47) & 4.42 & 8 & 4.40 & 5 & 4.38 & 6 & 4.34 & 3 & n.s. & 0 \\
\hline Left uncus (BA 28, 36) & 4.40 & 10 & 4.33 & 3 & n.s. & 0 & n.s. & 0 & n.s. & 0 \\
\hline total & & 151 & & 150 & & 178 & & 171 & & 139 \\
\hline
\end{tabular}

\section{Figures:}

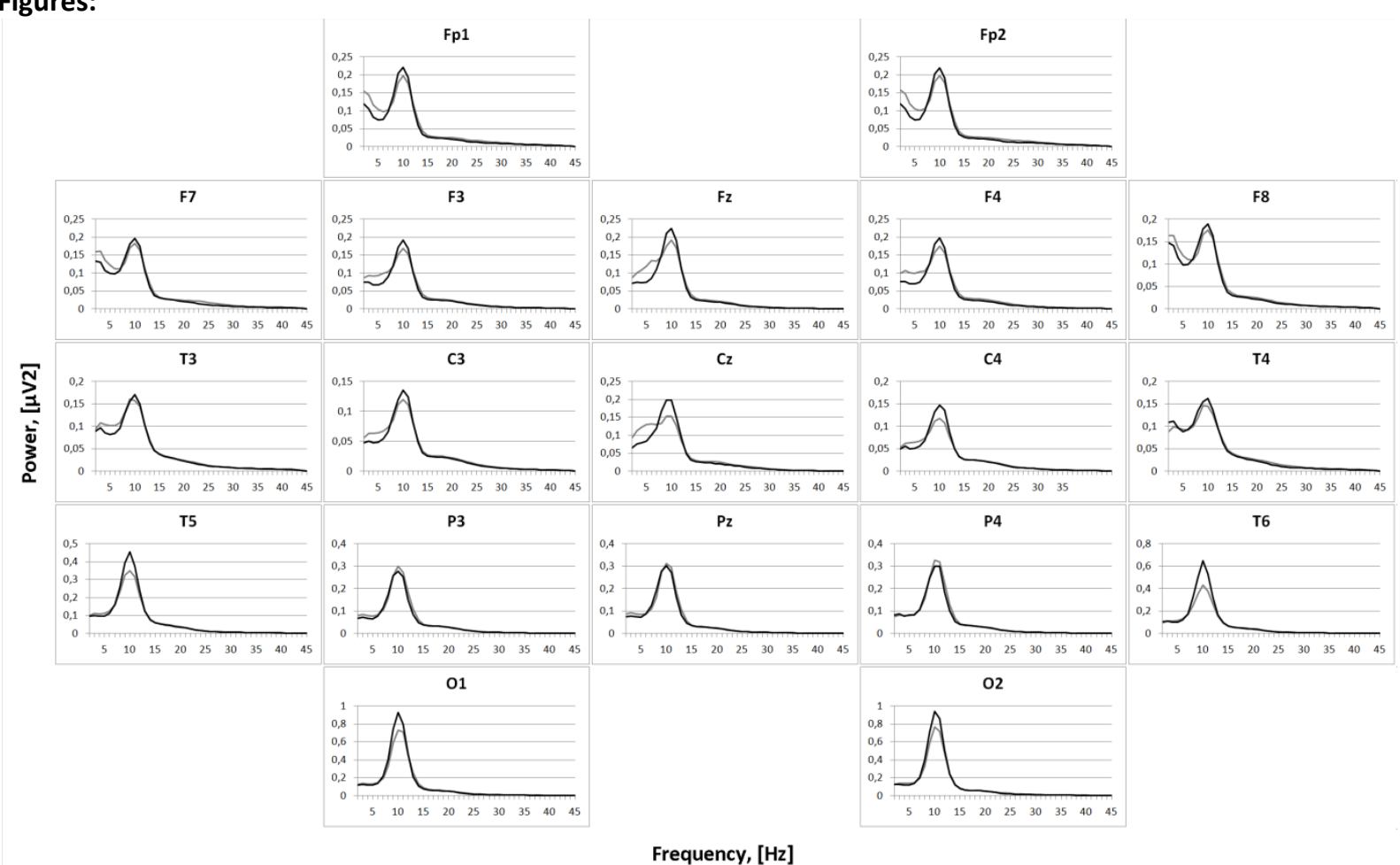

Figure 1: Mean normalized absolute power at all electrodes in OCD patients (grey line) and healthy controls (black line). 


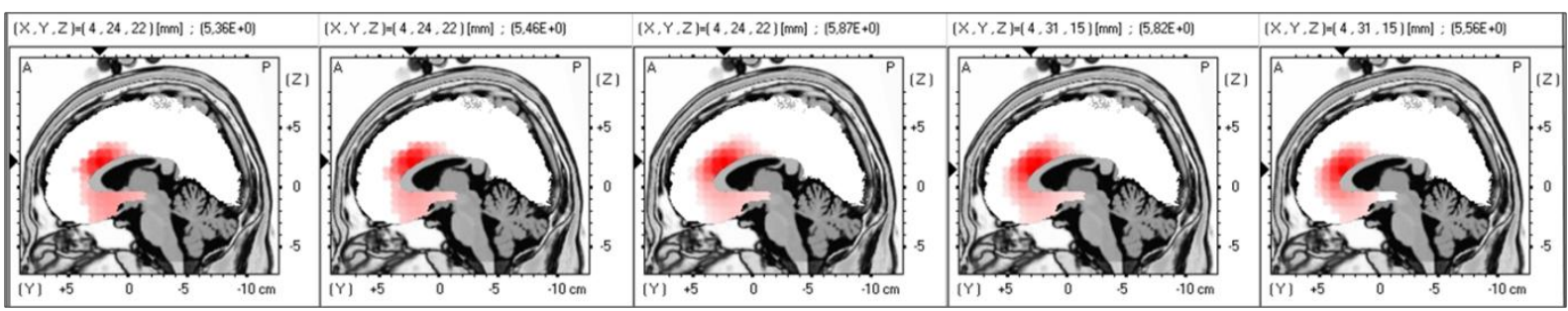

Figure 2: Sagittal view (left of the picture is front of the head) of absolute current density increase in OCD patients compared with controls at $2-6 \mathrm{~Hz}$. Each figure is sliced to its own $t$-value maximum $\left(t_{\max 2 \mathrm{~Hz}}=5.36, t_{\max 3 \mathrm{~Hz}}=5.46, t_{\max 4 \mathrm{~Hz}}=5.87, t_{\max 5 \mathrm{~Hz}}=5.82, t_{\max 6 \mathrm{~Hz}}=5.56\right)$. Figures show only the most significant voxels (the darker the red colour, the higher the $t$-value). For all significant voxels see Table 2 .

\section{sLORETA absolute power in controls and patients}

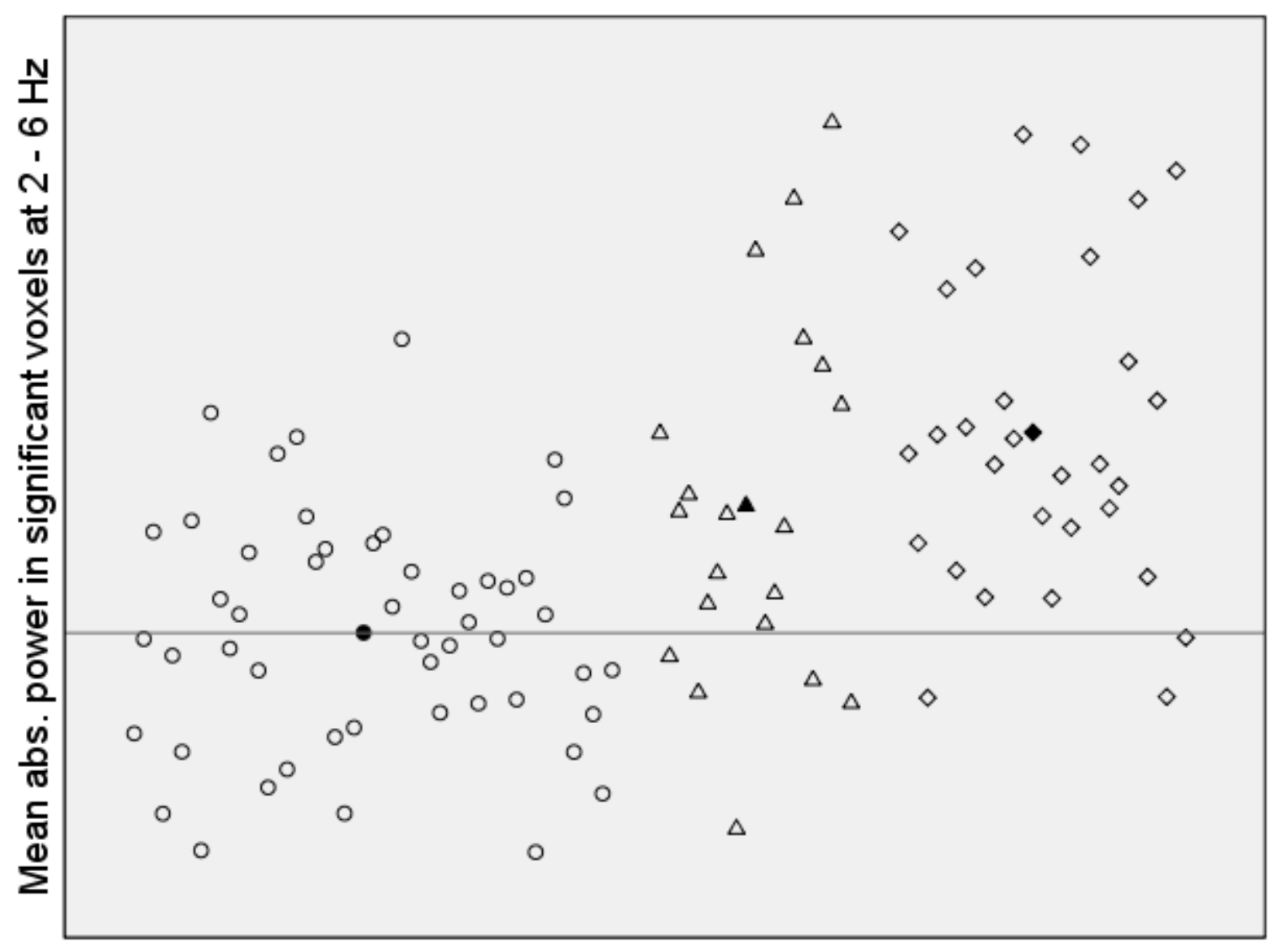

Controls - drug-free OCD - OCD medicated with SSRIs

Figure 3: Mean absolute current density at low frequencies $(2-6 \mathrm{~Hz})$ in controls (circles), drug-free (triangles) and medicated OCD (diamonds) patients. The filled elements indicate the mean current density value in each group. 




Figure 4: Seven independent components separated in the control group. The components are sorted according to explained variance and the images are sliced to their own maxima. From left to right, the inserts 1 to 7 show the axial (top of the picture is front of the head), sagittal (left of the picture is front of the head) and coronal (top of the picture is top of the head) view. The intensity of the red colour indicates the intensity of the signal contributing to the component (arbitrary unit and scaled to its own maximum for each component). Component 5 is also displayed on a three-dimensional cortical template.
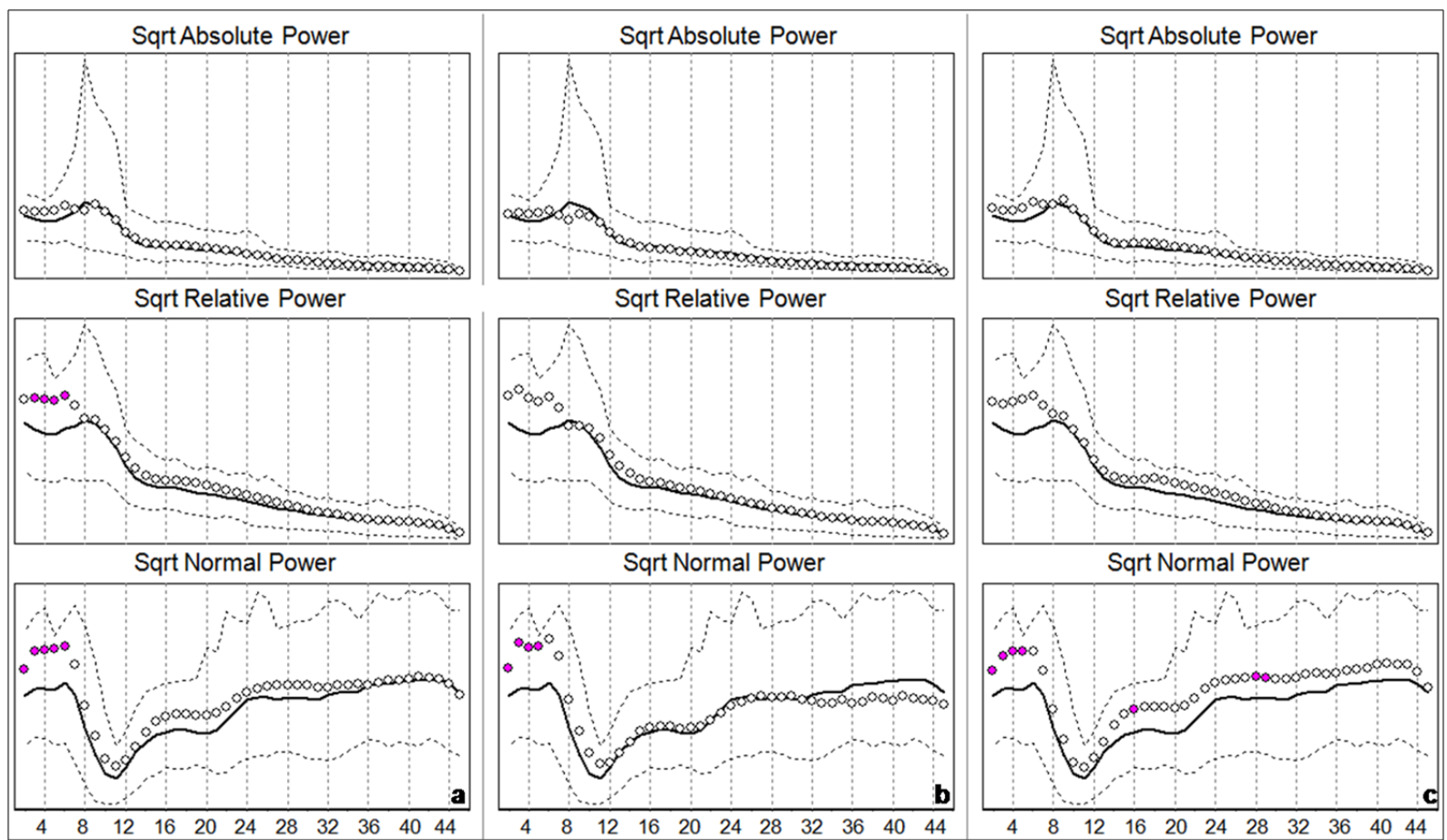

Figure 5: Absolute, relative and normal power (arbitrary units) of the $5^{\text {th }}$ component along frequencies in the range $2-45 \mathrm{~Hz}$. Comparison between OCD patients and controls (a), drug-free patients and controls (b) and medicated patients and controls (c). In each insert the solid and dotted lines indicate the mean and $95 \%$ confidence interval for the normal group, whereas the disks indicate the mean of the OCD group, with violet disks flagging significant power increase ( $<<0.05$, corrected). 